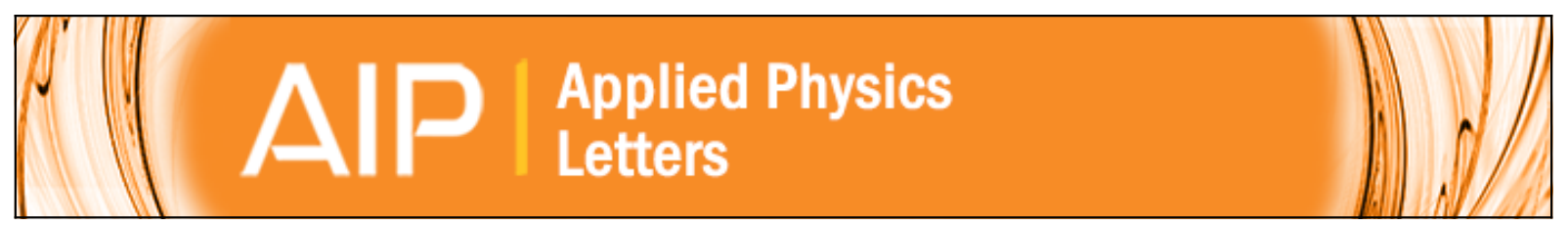

\title{
Influence of magnetic interactions between phases on the magnetocaloric effect of composites
}

C. Romero-Muñiz, V. Franco, and A. Conde

Citation: Applied Physics Letters 102, 082402 (2013); doi: 10.1063/1.4793663

View online: http://dx.doi.org/10.1063/1.4793663

View Table of Contents: http://scitation.aip.org/content/aip/journal/apl/102/8?ver=pdfcov

Published by the AIP Publishing

\section{Articles you may be interested in}

The magnetic phase diagram and large reversible room-temperature magnetocaloric effect in antiperovskite compounds $\mathrm{Zn} 1-\mathrm{x} \mathrm{Sn} \times \mathrm{CFe} 3(0 \leq \mathrm{x} \leq 1)$

J. Appl. Phys. 112, 063904 (2012); 10.1063/1.4752275

The influence of Si addition on the glass forming ability, magnetic and magnetocaloric properties of the Gd-Fe-Al glassy ribbons

J. Appl. Phys. 109, 07 A911 (2011); 10.1063/1.3540666

Influence of $\mathrm{Mn}$ on the magnetocaloric effect of nanoperm-type alloys

J. Appl. Phys. 108, 073921 (2010); 10.1063/1.3489990

Interstitial-nitrogen effect on phase transition and magnetocaloric effect in $\mathrm{Mn}(\mathrm{As}, \mathrm{Si})$ (invited)

J. Appl. Phys. 107, 09A938 (2010); 10.1063/1.3358617

Tunable magnetic regenerator alloys with a giant magnetocaloric effect for magnetic refrigeration from $\sim 20$ to $~$ $290 \mathrm{~K}$

Appl. Phys. Lett. 70, 3299 (1997); 10.1063/1.119206

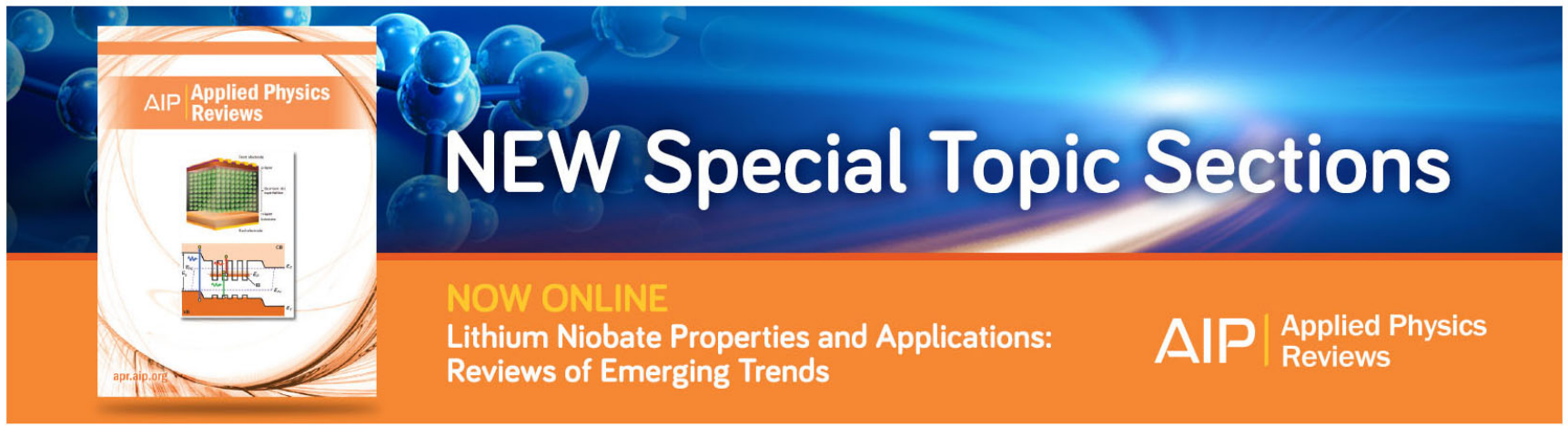




\title{
Influence of magnetic interactions between phases on the magnetocaloric effect of composites
}

\author{
C. Romero-Muñiz, ${ }^{\text {a) }}$ V. Franco, ${ }^{\text {b) }}$ and A. Conde \\ Dpto. Física de la Materia Condensada, ICMSE-CSIC, Universidad de Sevilla, P.O. Box 1065, \\ 41080-Sevilla, Spain
}

(Received 8 January 2013; accepted 14 February 2013; published online 26 February 2013)

\begin{abstract}
Magnetocaloric materials with coexisting magnetic phases appear either due to the phase coexistence in first order phase transitions, or due to the development of composites, which are known to enhance the refrigerant capacity and produce table-like magnetocaloric effect. However, interactions between phases are rarely considered. We have modeled the influence of interactions on the magnetocaloric effect of a biphasic composite by implementing a mean field model. Interactions shift the peak magnetic entropy change to higher temperatures than those of the pure phases and enhance the table-like character of the curves. Although there is no qualitative change of the magnetocaloric response of the composites due to interactions, the optimal fraction of phases which produces the largest enhancement of the refrigerant capacity is shifted to compositions richer in the low Curie temperature phase. This shift can be used to estimate the magnitude of the interactions in composites measured experimentally. (C) 2013 American Institute of Physics.
\end{abstract}

[http://dx.doi.org/10.1063/1.4793663]

In the recent years, research on the magnetic properties of materials is being progressively more focused on strategies to increase the energy efficiency of our society. ${ }^{1-3}$ Apart from the improvement of currently existing applications via the optimization of materials' properties (like the enhancement of soft and hard magnetic materials for their implementation in more efficient motors, transformers, and generators), there are new applications of magnetic materials, which can help us reach larger energy efficiency. One of them is magnetic refrigeration, associated to the magnetocaloric effect, which is gaining increasing scientific and technological attention. ${ }^{1,4-7}$ Taking into account that the largest electricity consumption in the residential market is related to refrigeration and air conditioning, the improvement of these devices via the use of magnetic technologies will translate into a large reduction in energy consumption.

The magnetocaloric effect (MCE), i.e., the reversible temperature change of a magnetic material upon the application/ removal of a magnetic field, is routinely used in research laboratories to achieve ultra-low temperatures. For operation closer to room temperature, magnetic refrigerators have to employ materials with a magnetic (or magneto-structural) phase transition in that temperature range, which gives rise to an abrupt temperature change of magnetization. And although magnetic refrigeration is not a commercial technology yet, current prototypes have shown that energy efficiency can be increased by $50 \%$ with respect to the optimal refrigerators based on the compression/expansion of gasses. If we add to the equation the absence of gasses responsible for greenhouse effect and ozone depletion, the reduced vibration and noise motivated by the lack of a large compressor, and the operation at any tilt angle, we find the arguments which foster research in magnetocaloric materials.

\footnotetext{
${ }^{a)}$ Present address: Dpto. Física Teórica de la Materia Condensada, Universidad Autónoma de Madrid, E-28049 Madrid, Spain.

b) Electronic mail: vfranco@us.es.
}

Since Brown proposed its application for this purpose in $1974,{ }^{8}$ the archetypical magnetocaloric material is $\mathrm{Gd}$, due to its large magnetic moment and its second order magnetic transition close to room temperature. Subsequently, the discovery of the giant magnetocaloric effect in $\mathrm{GdSiGe}^{9}$ shifted the interest to materials with a first order magneto-structural transition. This latter kind of materials exhibit a larger magnetic entropy change peak, $\Delta S_{M}^{p k}$, but the nature of the phase transition has inherent thermal hysteresis, which complicates its applicability in current prototypes. At the moment, most magnetic refrigerators use either Gd (the majority of them) or $\mathrm{LaFeSiH}$ as refrigerant materials, although the latter has the drawbacks of mechanical degradation after magnetic field cycling and splitting of the magnetic entropy change peaks due to instability of the hydride phase, issues which are driving further research to overcome them. ${ }^{7,10,11}$

In the study of the thermomagnetic transitions which give rise to the magnetocaloric effect, we have to consider two different scenarios. In the case of single phase materials with a second order phase transition, the continuous nature of the transition simplifies our study, in the sense that we do not need to consider coexisting magnetic phases and the description of the associated magnetocaloric effect can be done with the use of more or less simple equations of state. ${ }^{12}$ However, first order phase transition materials inherently present coexisting magnetic phases within the temperature and magnetic field ranges in which the transition takes place, which is a challenge for modeling the phenomena and seriously affects the dynamic response of the magnetic refrigerant material when it is embedded in a magnetic refrigerator. In fact, there are experimental observations which indicate that this coexistence of phases affect dynamic properties such as the magnetic resonance spectra. ${ }^{13}$ However, first order phase transition magnetocaloric materials are not the only kind of systems in which different magnetic phases coexist. It has been recently shown theoretically that composite materials constituted by different phases with a second order phase 
transition can exhibit greatly enhanced refrigerant capacities (RC), and table-like magnetocaloric effect. ${ }^{13}$ This has been subsequently confirmed by experimental results by different groups, ${ }^{14-16}$ finding an outstanding agreement between experimental results and predictions of the models. ${ }^{16}$ However, these studies of materials with different magnetic phases do not consider the possible effect of the interactions among the phases, which might alter the response of the materials. The agreement between experiment and the models which do not consider interactions might either be due to a much reduced value of magnetic interactions in the systems under study, or to a very limited influence of interactions on the response of the composite. In order to discern which the actual situation is, we have performed simulations of the thermomagnetic response of multiphase materials with different degrees of magnetic coupling between phases. Our simplifying approach, which enables us to extract the main influence of interactions between phases, has been to simulate a composite material formed from phases undergoing a second order phase transition and with interactions represented by the mean field approach proposed by Callen et al. ${ }^{17}$ Although the mean field simplification might not give a correct microscopic representation of the system, it allows us to reproduce the thermomagnetic response in the magnetic field range from saturation to remanence, ${ }^{18}$ which is the most relevant in magnetocaloric studies. It is worth mentioning that our approach is conceptually different from the case of superferromagnetic particles (an ensemble of nanoparticles which, in the absence of interactions, would be superparamagnetic). ${ }^{19-21}$ In that case, the interaction-free system would present a continuous decay of the magnetic entropy change and the appearance of the magnetocaloric peak at finite temperatures is due to the presence of interactions. In our case, the different phases of the composite already present ferro-paramagnetic transitions, with their own magnetic entropy change peaks. Nevertheless, in both cases, the approach to consider the interactions has been a mean field. It is worth mentioning that in our previous publications related to magnetocaloric effect and thermomagnetic phase transitions, we have stressed that the critical exponents of the phase transition do not necessarily have to be those of a mean field model, as indicated in our previous experimental results. ${ }^{12,22-24}$ Therefore, the exponent controlling the field dependence of the magnetic entropy change does not have to be $2 / 3$, as predicted by Osterreicher and Parker. ${ }^{25}$ However, the mean field approach that we are following in this case is different: we are representing the influence of the different phases of the composite sample on the behavior of the rest of the system by an effective field, which is proportional to the magnetization of the sample. This is also considered a mean field approach and is a first approximation to describing the influence of interactions. We, therefore, consider that the thermomagnetic properties of each of the non-interacting phases are described by the Arrott-Noakes equation of state, ${ }^{26}$ with critical exponents which are representative of typical soft magnetic amorphous alloys ${ }^{27,28}$ (not mean field exponents), while the interactions between phases are introduced into the model by a mean field approach.

In this work, we use the Arrott-Noakes equation of state ${ }^{26}$ to reproduce the magnetothermal response of each phase

$$
\left(\frac{H}{M}\right)^{1 / \gamma}=a\left(T-T_{c}\right)+b M^{1 / \beta},
$$

where $\beta$ and $\gamma$ are critical exponents, $T_{c}$ is the Curie temperature, and $a$ and $b$ are two fitting parameters of each phase, related to the initial susceptibility and saturation magnetization. This equation can be used to accurately reproduce the field and temperature dependence of the magnetic entropy change, $\Delta S_{M}$, of each phase in the environment of $T_{c}{ }^{28}$ The values of the different parameters used in this work correspond to those of a typical soft magnetic amorphous alloy: ${ }^{28} \mathrm{a}=1.228$ (Oe g/emu) ${ }^{1 / \gamma} \mathrm{K}^{-1} ; \mathrm{b}=7.7010^{-3} \mathrm{Oe}^{1 / \gamma}$ $(\mathrm{g} / \mathrm{emu})^{1 / \gamma+1 / \beta}$; and $\beta=0.4579$ and $\gamma=1.5593$. We fix the Curie temperature of one of the phases as $\mathrm{T}_{\mathrm{cA}}=370 \mathrm{~K}$ and for phase $\mathrm{B}$, we study the range $240 \mathrm{~K} \leq T_{c B}<T_{c A}$. In order to limit the number of free parameters in our model material, we have used the same values of the critical exponents for both phases. This simplification is supported by the fact that minor compositional changes-e.g., alloys from the same compositional series-do not alter the scaling behavior of their magnetocaloric effect, indicating that the critical exponents are not affected. ${ }^{27,29}$

Once Eq. (1) is used for calculating the field and temperature dependent magnetization curves of each phase, the magnetization of the non-interacting composite can be calculated using a rule of mixtures

$$
M=x M_{A}+(1-x) M_{B},
$$

where $x$ is the fraction of constituent A. The field and temperature dependent magnetic entropy change curves of the composite can be calculated from the numerical approximation to Maxwell relation

$$
\Delta S_{M}=\mu_{0} \int_{0}^{H}\left(\frac{\partial M}{\partial T}\right)_{H^{\prime}} d H^{\prime}
$$

in which the integral is replaced by a summation and the derivative is replaced by finite differences. The refrigerant capacity, a measure of the amount of heat which can be transferred between the cold and hot reservoirs, is calculated as

$$
R C=-\int_{T_{1}}^{T_{2}} \Delta S_{M} d T
$$

where the temperature span $\delta T_{F W H M}=T_{2}-T_{1}$ corresponds to the full width at half maximum of the $\Delta S_{M}$ peak.

In order to incorporate the interaction field in our model composite material, we have considered a mean field approach in which the effective field perceived by each phase corresponds to the addition of the applied field $H$ and the effect of the magnetization of the whole material weighted by the phenomenological constant $\lambda$, which is a measure of the strength of the interactions

$$
H_{e f f}=H+\lambda M
$$

The curves $M_{A, B}(\lambda=0)$ obtained from Eq. (1) can be transformed into $M_{A, B}(\lambda \neq 0)$ by means of Eq. (5), either with a self-consistent method, or making use of the fact that the 
thermomagnetic response of each of the phases, when plotted versus $H_{e f f}$, should coincide with that of the pure phase without interactions. ${ }^{18}$ As this latter procedure is faster and produces less noisy curves, it has been the one that we have used. The magnetic entropy change of the interacting composite is subsequently calculated using Eq. (3).

Figure 1 shows the temperature dependence of $\Delta S_{M}$ of the two constituent phases, along with the curve corresponding to a composite with different mean field strengths, $\lambda=0$, 10,50 , and $100 \mathrm{~g} \mathrm{~cm}^{-3}$ (as usual experimental results are expressed as magnetization per unit mass, instead of per unit volume, the interaction constant should have units of density). Interactions between phases have a twofold effect: on the one hand, both peaks shift to higher temperatures with increasing $\lambda$. On the other hand, the magnitude of the high temperature peak increases and the shape of the peak gets sharper. This combined effect makes the $\Delta S_{M}(T)$ curve enhance its table-like shape, which would be beneficial for Ericsson-type magnetic refrigerators. ${ }^{30}$ The justification for these changes is the following: for an interaction free system, once the Curie temperature of the low temperature phase is reached, the only part of the sample which is significantly contributing to the magnetic entropy change curve is the high temperature phase. However, by introducing the interactions in the system, the magnetization of the high Curie temperature phase acts as a driving force to increase the magnetization of the low $T_{C}$ phase, which causes a shift in the position of the peak towards higher temperatures. As expected, this shift is larger for the low temperature peak (the high $\mathrm{T}_{\mathrm{C}}$ phase is ferromagnetic and causes a larger influence on the other) than for the high temperature peak (the magnetization increase caused by the interactions is more discreet close to the high $\mathrm{T}_{\mathrm{C}}$ phase). This increased magnetization should also be responsible for the sharpening of the magnetic entropy change peaks, which is more relevant for temperatures close to the higher $\mathrm{T}_{\mathrm{C}}$.

For a systematic study of the influence of interactions on the composite, we have to take into account several factors that affect the MCE of composites, like the fraction of phases, the difference between the Curie temperature of two

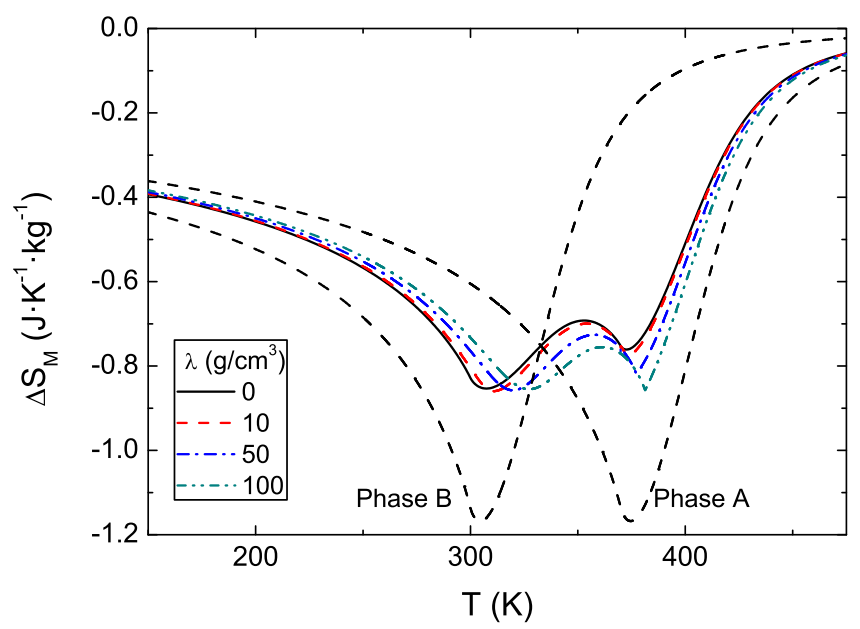

FIG. 1. Temperature dependence of the magnetic entropy change of two different phases and of a composite formed from them with $x=0.6$ and different interaction strengths. The applied field is $1.5 \mathrm{~T}$. phases $\Delta T_{c}=T_{c A}-T_{c B}$, the strength of the interactions, $\lambda$, and the maximum applied magnetic field $H$. As all parameters cannot be varied simultaneously, we have made to approaches: (a) fixing $\Delta T_{c}=130 \mathrm{~K}$ while increasing $H$ up to $10 \mathrm{~T}$ and (b) fixing the maximum applied field at $1.5 \mathrm{~T}$ while increasing $\Delta T_{c}$ from $0 \mathrm{~K}$ to $130 \mathrm{~K}$. In both cases, the entire phase fraction range $(0<x<1)$ has been examined in increments of 0.01 , and selected values of $\lambda$ have been chosen. In order to extract a single magnitude from each $\Delta S_{M}(T)$ curve, we have defined the refrigerant capacity improvement $(\mathrm{RCI})$, as the percentage improvement of the refrigerant capacity of the composite with respect to that of the majority constituent phase ${ }^{13}$

$$
R C I(\%)= \begin{cases}\frac{R C(x)-R C(x=0)}{R C(x=0)} \times 100 & \text { for } \quad x \leq 0.5 \\ \frac{R C(x)-R C(x=1)}{R C(x=1)} \times 100 & \text { for } \quad x>0.5 .\end{cases}
$$

Figure 2 shows the dependence of $R C I$ on (a) the fraction of phases and applied field; and (b) the fraction of phases and separation between their Curie temperatures. Both subfigures include the cases with $\lambda=0$ and $\lambda=100 \mathrm{~g} \mathrm{~cm}^{-3}$, to allow for a more visual analysis of the influence of the interactions. It is seen that although interactions between phases do not affect qualitatively the magnetocaloric behavior of biphasic composites, there are some differences in the details of the surfaces, like the maximum of $R C I$ which can be achieved and a shift of the $x, \Delta T_{c}$ and $H$ values which give rise to a particular value of $R C I$. To make more evident the different magnetocaloric responses for different interaction strengths, Figure 3 shows the changes of $R C I(x)$ for an applied field of $1.5 \mathrm{~T}$ and for different values of $\lambda$. In all cases, there exists an optimal composition, $x_{\text {opt }}$, which maximizes $R C I$, but increasing interactions shift it to lower $x$ (lower content of the high Curie temperature phase). This shift in $x_{\text {opt }}$ is a general behavior for all the studied values of applied field and for all $\Delta T_{c}$.

In order to compare these results emerging from numerical simulations with experimental data, we have studied the $R C I(x)$ curves recently obtained by Paticopoulos et al. ${ }^{16}$ for a biphasic composite. Even if the non-interacting model was able to accurately reproduce the main characteristics of the curves, there was an evident shift of the maximum $R C I$ to compositions with lower $x$ values, which is an indication of the existence of interactions between phases. In order to evaluate the magnitude of these interactions, we have measured these displacements for several values of applied fields and compared them with the displacement in composition predicted by our simulations. This information allows us to estimate that $\lambda \approx 50 \mathrm{~g} \mathrm{~cm}^{-3}$ for that particular experimental case. This corresponds to an interaction field ranging between 0.4 and $0.1 \mathrm{~T}$ in the temperature range between $T_{c A}$ and $T_{c B}$.

In conclusion, we have studied the influence of magnetic interactions in multiphase magnetic systems by means of a mean field model in a composite constituted by phases with a second order phase transition. Interactions enhance the table-like magnetocaloric response of the composite and shift the peak temperatures of the phases to higher temperatures. Interactions do not change qualitatively the dependence of the refrigerant capacity with field and fraction of 
(a)

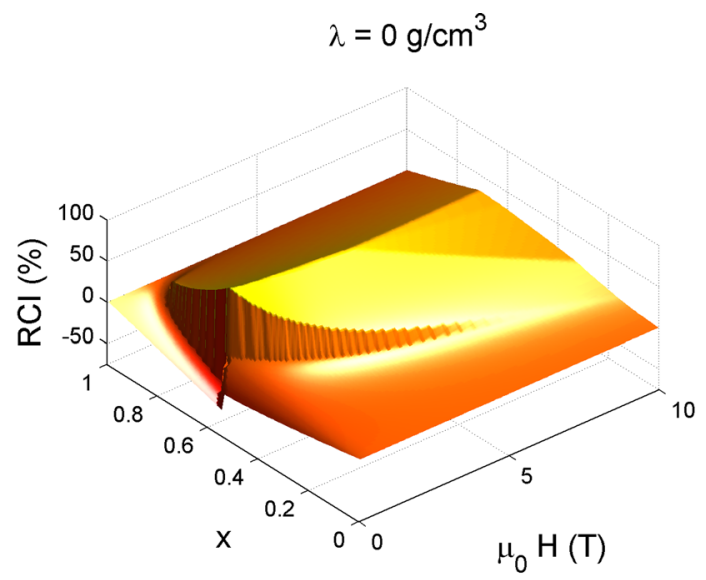

(b)

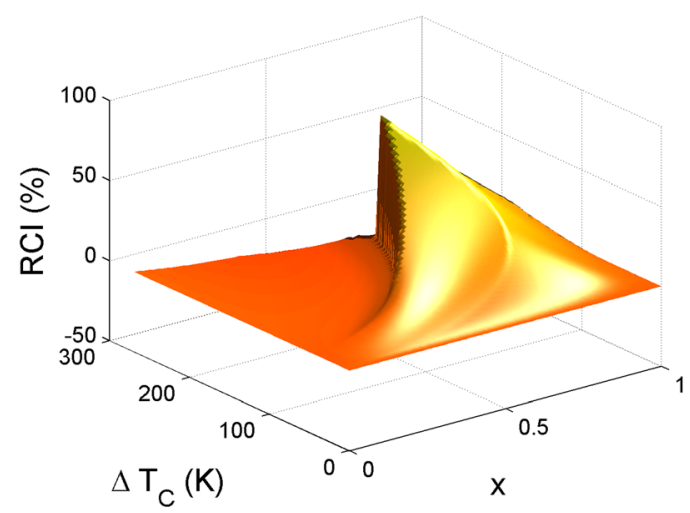

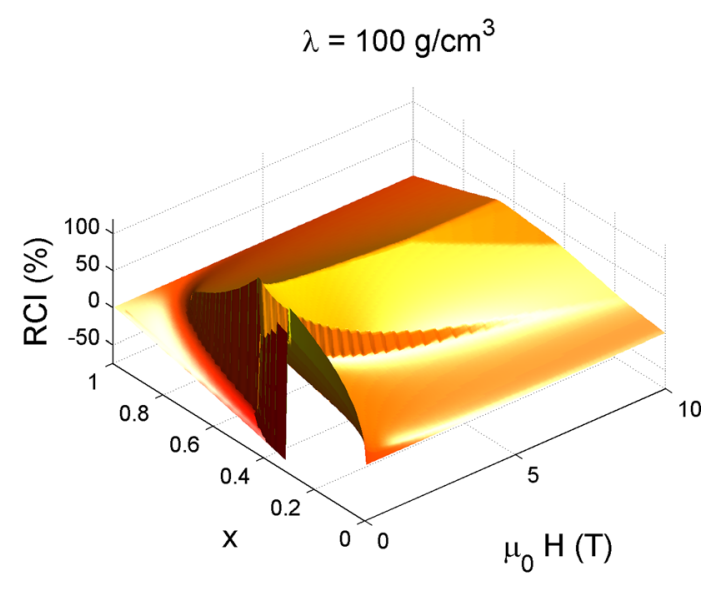

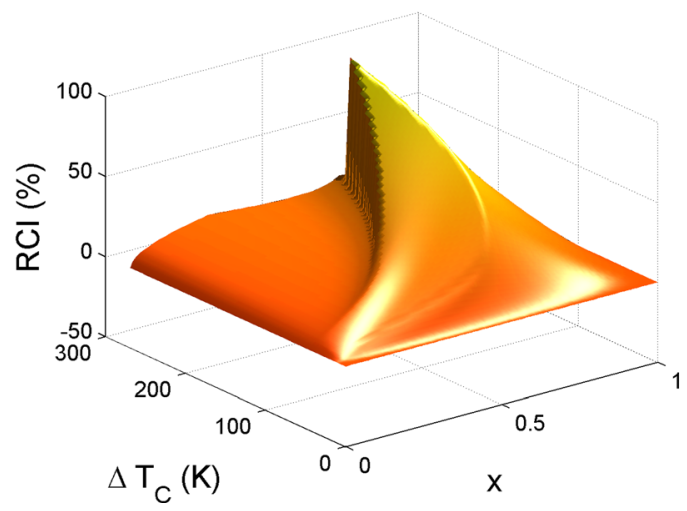

FIG. 2. Improvement of the refrigerant capacity of a composite as a function of (a) the fraction of phases and maximum applied field for a fixed $\Delta T_{c}=130 \mathrm{~K}$ and (b) the fraction of phases and separation between their Curie temperatures for a maximum applied field of $1.5 \mathrm{~T}$. Figures on the left are for a noninteracting composite, while figures on the right correspond to $\lambda=100 \mathrm{~g} \mathrm{~cm}^{-3}$.

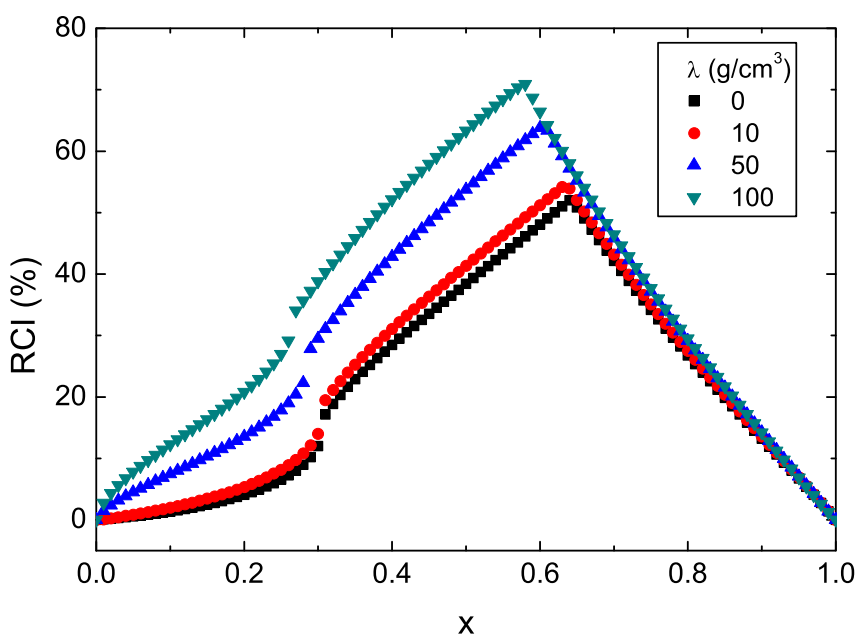

FIG. 3. Influence of interaction strength on compositional dependence of the improvement of the refrigerant capacity for a maximum applied field of $1.5 \mathrm{~T}$.

phases, existing optimal compositions for which the refrigerant capacity is largely enhanced with respect to that of the constituent phases. However, with increasing interaction between phases, the optimal $x$ is shifted to composites richer in the low Curie temperature phase. This shift can be used to estimate the mean field interaction parameter of experimentally measured composites. These results can, on one hand, let us design in a more accurate way the fractions of phases of a multiphase composite needed to obtain table-like magnetocaloric effect with enhanced refrigerant capacity. On the other hand, a similar procedure (with appropriate modifications) can be used to gain insight into the influence of interphase interactions in first order phase transition materials, for which the coexistence of magnetic phases is inherent to the nature of the transition.

This work was supported by the Spanish Ministry of Science and Innovation and EU FEDER (Project MAT 2010-20537), the PAI of the Regional Government of Andalucía (Project P10-FQM-6462), and the United States Office of Naval Research (Project N00014-11-1-0311).

${ }^{1}$ O. Gutfleisch, M. A. Willard, E. Bruck, C. H. Chen, S. G. Sankar, and J. P. Liu, Adv. Mater. 23, 821 (2011).

${ }^{2}$ V. Franco and O. Gutfleisch, JOM 64, 750 (2012).

${ }^{3}$ O. Gutfleisch and V. Franco, Scr. Mater. 67, 521 (2012).

${ }^{4}$ K. A. Gschneidner and V. K. Pecharsky, Ann. Rev. Mater. Sci. 30, 387 (2000).

${ }^{5}$ E. Bruck, J. Phys. D: Appl. Phys. 38, R381 (2005).

${ }^{6}$ B. G. Shen, J. R. Sun, F. X. Hu, H. W. Zhang, and Z. H. Cheng, Adv. Mater. 21, 4545 (2009).

${ }^{7}$ V. Franco, J. S. Blázquez, B. Ingale, and A. Conde, Ann. Rev. Mater. Res. 42, 305 (2012).

${ }^{8}$ G. V. Brown, J. Appl. Phys. 47, 3673 (1976).

${ }^{9}$ V. K. Pecharsky and K. A. Gschneidner, Phys. Rev. Lett. 78, 4494 (1997).

${ }^{10}$ J. Lyubina, R. Schafer, N. Martin, L. Schultz, and O. Gutfleisch, Adv. Mater. 22, 3735 (2010).

${ }^{11}$ J. Lyubina, U. Hannemann, M. P. Ryan, and L. F. Cohen, Adv. Mater. 24, 2042 (2012).

${ }^{12}$ V. Franco and A. Conde, Int. J. Refrig. 33, 465 (2010).

${ }^{13}$ R. Caballero-Flores, V. Franco, A. Conde, K. E. Knipling, and M. A. Willard, Appl. Phys. Lett. 98, 102505 (2011). 
${ }^{14}$ A. Chaturvedi, S. Stefanoski, M. H. Phan, G. S. Nolas, and H. Srikanth, Appl. Phys. Lett. 99, 162513 (2011).

${ }^{15}$ P. Alvarez, J. L. S. Llamazares, P. Gorria, and J. A. Blanco, Appl. Phys. Lett. 99, 232501 (2011).

${ }^{16}$ S. C. Paticopoulos, R. Caballero-Flores, V. Franco, J. S. Blazquez, A. Conde, K. E. Knipling, and M. A. Willard, Solid State Commun. 152, 1590 (2012).

${ }^{17}$ E. Callen, Y. J. Liu, and J. R. Cullen, Phys. Rev. B 16, 263 (1977).

${ }^{18}$ V. Franco and A. Conde, Appl. Phys. Lett. 74, 3875 (1999).

${ }^{19}$ L. H. Bennett, R. D. McMichael, L. J. Swartzendruber, R. D. Shull, and R. E. Watson, J. Magn. Magn. Mater. 104-107, 1094 (1992).

${ }^{20}$ S. Morup, Hyperfine Interact. 90, 171 (1994).

${ }^{21}$ M. S. Pedersen, S. Morup, S. Linderoth, C. Johansson, and M. Hanson, J. Phys. Condens. Matter 9, 7173 (1997).
${ }^{22}$ V. Franco, J. S. Blazquez, and A. Conde, Appl. Phys. Lett. 89, 222512 (2006).

${ }^{23}$ V. Franco, A. Conde, V. K. Pecharsky, and K. A. Gschneidner, EPL 79, 47009 (2007).

${ }^{24}$ V. Franco, A. Conde, J. M. Romero-Enrique, and J. S. Blazquez, J. Phys. Condens. Matter 20, 285207 (2008).

${ }^{25}$ H. Oesterreicher and F. T. Parker, J. Appl. Phys. 55, 4334 (1984).

${ }^{26}$ A. Arrott and J. E. Noakes, Phys. Rev. Lett. 19, 786 (1967).

${ }^{27}$ V. Franco, J. S. Blazquez, M. Millan, J. M. Borrego, C. F. Conde, and A. Conde, J. Appl. Phys. 101, 09C503 (2007).

${ }^{28}$ V. Franco, A. Conde, and L. F. Kiss, J. Appl. Phys. 104, 033903 (2008).

${ }^{29}$ V. Franco, C. F. Conde, J. S. Blazquez, A. Conde, P. Svec, D. Janickovic, and L. F. Kiss, J. Appl. Phys. 101, 093903 (2007).

${ }^{30}$ A. Smaili and R. Chahine, J. Appl. Phys. 81, 824 (1997). 